\title{
Report of the European Respiratory Society/European Cystic Fibrosis Society task force on the care of adults with cystic fibrosis
}

\author{
J. Stuart Elborn ${ }^{1}$, Scott C. Bell ${ }^{2}$, Susan L. Madge ${ }^{3}$, Pierre-Regis Burgel ${ }^{4}$, \\ Carlo Castellani ${ }^{5}$, Steven Conway ${ }^{6}$, Karleen De Rijcke $^{7}$, Birgit Dembski, \\ Pavel Drevinek ${ }^{9}$, Harry G.M. Heijerman ${ }^{10}$, J. Alistair Innes ${ }^{11}$, Anders Lindblad ${ }^{12}$, \\ Bruce Marshall ${ }^{13}$, Hanne V. Olesen ${ }^{14}$, Andreas L. Reimann ${ }^{15}$, Ampara Solé ${ }^{16}$, \\ Laura Viviani ${ }^{17}$, Thomas O.F. Wagner ${ }^{18}$, Tobias Welte $^{19}$ and Francesco Blasi ${ }^{20}$
}

Affiliations: 'School of Medicine, Dentistry and Biomedical Sciences, Queen's University of Belfast, Belfast, UK. ${ }^{2}$ Dept of Thoracic Medicine, The Prince Charles Hospital and QIMR Berghofer Medical Research Institute, Brisbane, Australia. ${ }^{3}$ Dept of Respiratory Medicine, Royal Brompton Hospital, London, UK. ${ }^{4}$ Dept of Respiratory Medicine, Cochin Hospital, Assistance Publique Hôpitaux de Paris and Université Paris Descartes, Sorbonne Paris Cité, Paris, France. ${ }^{5}$ Cystic Fibrosis Center, Azienda Ospedaliera Universitaria Integrata, Verona, Italy. ${ }^{6}$ Paediatric and Adult CF Units, Leeds Teaching Hospitals Trust, Leeds, UK. ${ }^{7}$ Cystic Fibrosis Europe, Brussels, Belgium. ${ }^{8}$ Mukoviszidose eV, Berlin, Germany. ${ }^{9}$ Dept of Medical Microbiology, Motol University Hospital and 2nd Faculty of Medicine, Charles University, Prague, Czech Republic. ${ }^{10} \mathrm{Haga}$ Teaching Hospital, Dept of Pulmonology and Cystic Fibrosis, The Hague, The Netherlands. ${ }^{11}$ Scottish Adult Cystic Fibrosis Service, Western General Hospital, Edinburgh, UK. ${ }^{12}$ Dept of Paediatrics, Queen Silvia Children's Hospital, Sahlgrenska University Hospital, Gothenburg, Sweden. ${ }^{13}$ Cystic Fibrosis Foundation, Bethesda, MD, USA. ${ }^{14} \mathrm{CF}$ Centre, Aarhus University Hospital, Aarhus, Denmark. ${ }^{15}$ Mukoviszidose Institut gGmbH, Bonn, Germany. ${ }^{16}$ Lung Transplant and Adult Cystic Fibrosis, Dept of Pneumology, University Hospital la FE, Valencia, Spain. ${ }^{17}$ Dipartimento di Scienze Cliniche e di Comunità, Università degli Studi di Milano, Milan, Italy. ${ }^{18}$ Christiane Herzog CF Centre, Dept of Respiratory Diseases, Goethe University Frankfurt am Main, Frankfurt am Main, Germany. ${ }^{19}$ Dept of Respiratory Medicine, Hannover Medical School, Hanover, Germany. ${ }^{20}$ Dept of Pathophysiology and Transplantation, University of Milan, IRCCS Fondazione Cà Granda, Milan, Italy.

\section{Correspondence:}

F. Blasi, Dept of Pathophysiology and Transplantation, University of Milan, IRCCS Fondazione Cà Granda, Via Francesco Sforza 35, 20122 Milan, Italy. E-mail: francesco.blasiđunimi.it

J.S. Elborn, School of Medicine, Dentistry and Biomedical Sciences; Queen's University Belfast, Room G.16, Health Sciences Building, 97 Lisburn Road, Belfast, BT9 7AE, UK. E-mail: s.elbornवqub.ac.uk

ABSTRACT The improved survival in people with cystic fibrosis has led to an increasing number of patients reaching adulthood. This trend is likely to be maintained over the next decades, suggesting a need to increase the number of centres with expertise in the management of adult patients with cystic fibrosis. These centres should be capable of delivering multidisciplinary care addressing the complexity of the disease, in addition to addressing the psychological burden on patients and their families. Further issues that require attention are organ transplantation and end of life management.

Lung disease in adults with cystic fibrosis drives most of the clinical care requirements, and major lifethreatening complications, such as respiratory infection, respiratory failure, pneumothorax and haemoptysis, and the management of lung transplantation require expertise from trained respiratory physicians. The taskforce therefore strongly reccommends that medical leadership in multidisciplinary adult teams should be attributed to a respiratory physician adequately trained in cystic fibrosis management.

The task force suggests the implementation of a core curriculum for trainees in adult respiratory medicine and the selection and accreditation of training centres that deliver postgraduate training to the standards of the HERMES programme.

@ERSpublications

Respiratory physicians, trained in adult CF management, should lead multidisciplinary teams taking care of patients http://ow.ly/SgRgj 


\section{Introduction}

The predicted median survival for people with cystic fibrosis (CF) has increased in an almost linear fashion over the past five decades [1-3]. In the 1940s to 1960s CF was usually fatal in early childhood because of the consequences of recurrent pulmonary infection and malnutrition due to pancreatic failure and subsequent mal-digestion of nutrients. Initial improvements in care included the introduction of pancreatic enzyme replacement therapy (PERT) in the 1960s and enteric coated enzymes in the early 1980s to treat pancreatic insufficiency in combination with a high calorie, high fat diet. Systemic antibiotic therapy for lung infection, initially for Staphylococcus aureus and subsequently against Gram-negative organisms such as Pseudomonas aeruginosa and the development and use of inhaled antibiotic therapies over successive decades have been important therapeutic developments [4-6]. Advances in antibiotic therapy, the introduction of mucolytics including dornase alpha (Pulmozyme), hypertonic saline and more effective methods of airway clearance have contributed to the major therapeutic advances from the 1970s to the current era [7]. These and other supportive therapies for complications of CF, such as allergic bronchopulmonary aspergillosis (ABPA), CF-related diabetes mellitus, liver disease, bone disease and sinus disease, have been delivered by multidisciplinary teams in CF centres where expertise has been developed, retained and shared to ensure optimal management for people with CF. Centralised care with dedicated CF teams has become and remains the cornerstone of care delivery in CF $[3,8]$.

As survival has increased, the number of adults with CF is rapidly increasing, as the majority of children now live into adulthood. In countries with well-developed CF care there are few deaths in children; the number of people over the age of 18 years with $\mathrm{CF}$ is now either equal to or greater than the number under the age of 18 years (table 1). Registries in Europe, the USA and Canada now demonstrate that median survival for CF is around 40 years (table 1 ) and the median age at death is around 30 years. While there is progressive improvement in survival these measures are sensitive to year-to-year changes. There are challenges with completeness of case ascertainment and variability in the methods used for calculations across/between registries and so these data should be interpreted with caution.

This increase in the number of adults with CF and their likely further increased survival has shifted the balance of numbers in CF medicine to a majority of adults. Improvements in the health of children and the widespread introduction of newborn screening is changing the priorities of care to a preventative orientation. Optimal paediatric care seeks to prevent chronic infection, malnutrition and nutritional deficiencies, and screen for early complications of CF. While this emphasis on prevention of disease progression and complications remains in adult care, the morbidity associated with recurrent pulmonary exacerbations often requiring hospitalisation, under-nutrition and the many systemic complications occurring in CF are more prominent. Therefore, there is a requirement to deliver expert and responsive care for adults with CF for all aspects of disease $[3,9]$.

In light of these developments this task force has addressed a number of questions relating to adult CF care to signpost the priorities for the provision of optimal management of adult CF over the next 15 years. The task force has reviewed the current provision of care for adults with CF across Europe, estimated the likely number of adults by country (where sufficient registry data are available) and, where possible, made manpower projections for the numbers and skills of CF physicians and other healthcare professionals that will be required to provide optimal care as outlined by the recently published European Cystic Fibrosis Society (ECFS) standards of care [8]. Furthermore, we have reviewed the requirements for a curriculum for training physicians in CF care. This will ensure that CF care physicians have appropriate training in the management of adults with CF including the leadership qualities necessary to lead a CF multidisciplinary care team.

\section{Taskforce methodology}

The task force was appointed by the co-chairs from the membership of the European Respiratory Society (ERS) and ECFS. Members of the group were identified as opinion leaders in the field who were delivering care to people with CF. Individuals responsible for ECFS standards of care were included and international representation came from Australia and USA. National CF organisations and CF Europe provided a patient contribution. The area of adult care has very little primary literature and the task force wished to include description studies from a wide range of long term conditions outside of CF where transition from paediatric care is an issue. The literature was reviewed by the co-chairs who provided a first draft of the key areas of discussion. Three face-to-face meetings were organised to discuss the literature and contents. In the final meeting over 2 days a consensus position was agreed. The task force ensured all available literature had been considered and that any areas of controversy were discussed and a consensus decision was made on all statements.

Conflict of interest: Disclosures can be found alongside the online version of this article at erj.ersjournals.com 
TABLE 1 Reported median survival and numbers of children and adults with cystic fibrosis from selected registries

\begin{tabular}{|c|c|c|c|c|c|}
\hline Country & $\begin{array}{l}\text { Year of annual } \\
\text { report }\end{array}$ & $\begin{array}{l}\text { Median age } \\
\text { at death years }\end{array}$ & $\begin{array}{l}\text { Median predicted } \\
\text { age of survival years }\end{array}$ & $\begin{array}{l}\text { Percentage of adult } \\
\text { patients }(\geqslant 18 \text { years) }\end{array}$ & $\begin{array}{l}\text { Total number } \\
\text { of patients }\end{array}$ \\
\hline Belgium & $2011^{\#}$ & 33 & NA & 54.7 & 1171 \\
\hline France & 2012 & 28 & NA & 47.2 & $6196^{\pi}$ \\
\hline Germany & 2012 & 32 & NA & 52.4 & 5242 \\
\hline UK & 2012 & 28 & 43.5 & $57.6^{+}$ & $10078^{\S}$ \\
\hline USA & 2012 & NA & 41.1 & 49.1 & 27804 \\
\hline
\end{tabular}

\section{Forecasting of numbers of adults with CF}

The progressive increase in median survival will result in a significant increase in numbers of adults with CF over the next decade [10]. It is still unclear when the improvements in survival will plateau. The very encouraging results from corrector and corrector/potentiator combination therapy indicates that cystic fibrosis transmembrane conductance regulator (CFTR) is a "druggable" target [11-13]. If started early in life, these treatments could prevent the development of severe lung disease and postpone morbidity and result in further improvements in survival in the future [14].

Using data from the ECFS patient registry, the number of adults with CF is forecasted to increase by $75 \%$ between 2010 and 2025 [10]. This forecast is likely to be conservative as it does not account for the importance of potentiator and corrector therapies. These significant increases in numbers will require significant expansion of services for adults in countries with well-developed paediatric centres. To meet the needs of these increasing numbers of patients a proportional increase in CF multidisciplinary teams will be required, assuming that a CF centre provides care for 250 people with CF. It is likely that this will create significant challenges for healthcare funding agencies and hospital facilities to ensure that sufficient resources are available to develop and sustain adult CF teams.

\section{Manpower planning}

The anticipated increase in the number of patients will require the training of more physicians (and healthcare team members) in Europe to deliver medical care and lead multidisciplinary teams in this field. Training requires fundamental understanding of the lung disease in CF and other disease manifestations, such as diabetes, liver disease, bone disease, sino-nasal disease, kidney disease, joint disease and mental health issues. In addition, there needs to be a strong understanding of infertility, pregnancy, nutrition and other complications of CF.

National training schemes in pulmonology should ensure all trainees have a basic understanding of CF lung disease [3]. Consequently programmes to encourage and facilitate the training of the next generation of CF specialist physicians are required to meet the imminent needs of the rapidly growing group of adult CF patients. Ideally, these should include at least 1 year of specialist training in a well-established adult CF centre.

In order to develop a specific curriculum for respiratory physicians taking care of adult patients with CF the task force will submit a proposal for a HERMES task force to the ERS.

\section{Complexity of adult CF care}

In parallel with an increased population of adults with CF, complexity of care is steadily growing. Well-established complications (e.g. CF-related diabetes, massive haemoptysis, pneumothorax) are more common in adult patients and the treatment of infections caused by multi-resistant micro-organisms is made more complex by frequent adverse reactions to drugs, toxicity (including renal insufficiency and vestibular/ototoxicity) $[15,16]$ and problems associated with vascular access. Other less well-defined but emerging complications may well become common in the future care for adults living into their fourth decade and beyond. Gastro-intestinal cancers are more common in adults with CF, with the most prevalent being colorectal cancers $[17,18]$. The metabolic consequences of being overweight or obese with $\mathrm{CF}$ are unclear; however, as adults live longer and potentiator and corrector therapy increases body mass index, problems with obesity may become an issue [19]. Hyperlipidaemia in the adult CF population has recently been recognised and may add to risk in the future $[3,9]$. Long term systemic inflammation may 
be associated with cardiovascular complications and early evidence of increased arterial stiffness has been reported in children with CF [20]. The range and frequency of complications are likely to expand in the coming years [9].

\section{Framework for adult CF centres}

The optimal framework for adult CF centres is described in detail in the ECFS standard of care framework for the CF centre [8] which provides detailed information regarding centre staffing (table 2) for the CF multidisciplinary team and the skills required for delivery of multidisciplinary care in this context. This framework describes the differences between paediatric and adult centres for numbers of staff required, reflecting the significantly higher morbidity in patients attending adult centres. The framework also emphasises a number of key areas including transition from paediatric to adult services, specific adult issues relating to disease progression and life events unrelated to $\mathrm{CF}$, and transplantation with end-of-life issues. Within the ECFS standards of care for CF, best practice for care of people with CF is addressed through a series of questions outlining key therapies required to deliver optimal treatment in CF [21]. Finally, the ECFS standard of care for CF also deals with quality improvement and management in CF care to ensure the best care possible is delivered in CF centres [22].

\section{Specific management considerations for adults with CF Transition from paediatric to adult care}

The transition process from childhood to adulthood, including clinical care, is very important in the effective delivery of services for adults [23]. Ideally, all adult CF centres should have a close working relationship with the paediatric centres who regularly refer patients. Adolescence is a vulnerable period due to physiological, developmental and psychosocial changes. This stage of life is frequently associated with poor treatment adherence and decline in respiratory function and nutritional status [24-26]. Transition works best when there is close integration between paediatric and adult centres and the process directly involves the adolescents with CF and their families in the process [27]. This can be achieved by transition clinics that share between paediatric and adult teams prior to transfer to adult centres for full time care. Other models of care involve a single transfer clinic where adolescents and their families meet the adult care team at the last clinic visit to the paediatric services. Referral by letter only may be required in some circumstances but is considered suboptimal. Multidisciplinary team leadership is important and preparation by specialist nurses, psychologists and social workers is particularly helpful. The young person with CF should be central to this process and their needs for education, relationships and employment are as important as their medical care. The changing role of parents, siblings and friends during transition are also important to consider. Approaches for optimal transition are detailed in the ECFS standards of care and are well described in important disease-specific and generic guidelines [8, 21, 22, 25-27].

What are the main psychosocial issues confronting adults with CF?

Adults with CF have many psychosocial challenges. Family relationships, employment and education are key issues; however, adherence to treatment, concealment of disease and addressing needs for reduced time spent either in work or in education are also important considerations. Anxiety and depression are also highly prevalent in adults with CF [28]. Relationships and issues of fertility and reproduction are challenging areas for both women and men with CF requiring specialist knowledge and skilled counselling

TABLE 2 Whole-time equivalents per clinic size: adult patients

\begin{tabular}{lccc} 
The multidisciplinary team & $\mathbf{1 0 0}$ patients & $\mathbf{1 5 0}$ patients & $\mathbf{2 5 0}$ patients \\
\hline Consultant 1 & 0.5 & 1 & 1 \\
Consultant 2 & 0.3 & 0.5 & 1 \\
Consultant 3 & & & 0.5 \\
Staff grade/fellow & 0.5 & 1 & 1 \\
Specialist registrar & 0.4 & 0.8 & 1 \\
Specialist nurse & 2 & 3 & 5 \\
Physiotherapist & 2 & 4 & 6 \\
Dietician & 0.5 & 1 & 2 \\
Clinical psychologist & 0.5 & 1 & 2 \\
Social worker & 0.5 & 1 & 2 \\
Pharmacist & 0.5 & 1 & 1 \\
Secretary & 0.5 & 0.8 & 2 \\
Database coordinator & 0.4 & & 1 \\
\hline
\end{tabular}


[29]. Managing fertility and pregnancy with CF requires expertise and close collaboration and coordination with obstetric teams who regularly manage complex pregnancies. Other important issues include new diagnoses, such as diabetes, the impact of deteriorating health on family relationships and careers, respiratory failure, oxygen requirement and transplantation, all of which require specific expertise [30]. Other lifestyle issues, such as the use of tobacco, alcohol and recreational drugs, may also complicate care of $\mathrm{CF}$ care and require expertise in their management.

\section{Adherence}

Adherence to a complex treatment regimen is time consuming, intrusive and an often overwhelming responsibility [31, 32]. Practical and psychosocial support has to be provided in parallel [33]. Adherence changes with age and stage of disease; therefore, multidisciplinary teams must be aware of each patient's health status and ability to manage change when adding new therapies [25]. All care providers should help support patients. Specific psychological therapies (e.g. cognitive behavioural therapy, motivational interviewing) are often the most useful when non-adherence interferes with health status. The clinical team consulting with psychologists and, where appropriate, psychiatric colleagues, should assess each patient for the most appropriate approach. Psychotherapeutic options are described in a recent consensus report on the diagnosis and management of anxiety and depression in people with CF [34].

\section{Psychosocial support}

Practitioners with specific skills in supporting young people through chronic disease in parallel with major life events associated with adolescence and young adulthood are important in adult CF centres [35]. For an individual with $\mathrm{CF}$ it is vital that the interaction of normal life events, the psychological consequences of their disease and coping with complex therapies is considered holistically and managed appropriately [36, 37]. Specific disciplines involved may include clinical psychologists, social workers and/or specialist nurses [34].

\section{Late diagnosis of CF and CFTR-related disorders}

$\mathrm{CF}$ can be diagnosed later in life and awareness of this possible scenario is important for all clinicians [38]. The appropriate diagnostic tests of sweat chloride concentration and mutation analysis should be undertaken. Some late-diagnosed patients have mutations associated with a less severe phenotype [39]. All patients presenting with chronic pulmonary symptoms of cough and sputum, who have bronchiectasis on a chest computed tomography scan, and $P$. aeruginosa or $S$. aureus infection in adulthood should be considered as having a potential diagnosis of CF. People with CF diagnosed in adulthood often find it difficult to come to terms with the diagnosis and its implications and will require psychosocial support and ongoing education [40].

A group of conditions called CFTR-related disorders are frequently identified in adulthood [41]. All respiratory physicians, and physicians from other disciplines, such as gastroenterologists, otolaryngologists and fertility experts, should be aware of this potential diagnosis. CFTR-related disorders have been defined by a previous consensus committee as "a clinical entity associated with CFTR dysfunction that does not fulfil diagnostic criteria for CF." Patients presenting with obstructive azoospermia, acute recurrent or chronic pancreatitis and diffuse bronchiectasis should be considered as potential patients in this disease spectrum. Patients will usually have sweat chloride concentrations between 30 and $60 \mathrm{mmol} \cdot \mathrm{L}^{-1}$, may only demonstrate one CFTR mutation on limited CF diagnostic panels and, in many cases, will only have single organ involvement. Diagnosis of these conditions is important and appropriate genotyping (e.g. CFTR gene sequencing) should be considered so that suitable treatment can be offered. In some larger $\mathrm{CF}$ centres specific clinics have been established for patients with CFTR-related disease, as some of these patients may develop further symptoms allowing a definitive diagnosis of CF to be made.

\section{Lung transplantation}

Lung transplantation remains an important consideration for end stage respiratory disease in people with CF [40]. All adult CF centres should have ready access to a lung transplantation service and, when required, liver and renal transplantation services. The criteria for referral and the management of lung transplantation have recently been reviewed [41]. CF adult centres should have knowledge of, and have access to, supportive ventilation, including extracorporeal membrane oxygenation (ECMO) or extracorporeal life support (ECLS) [42,43]. The adult with CF should remain under the primary care of the transplantation team after a successful transplant and there should be shared care for pulmonary and non-pulmonary complications with the CF team. Primary care could be progressively handed over to $\mathrm{CF}$ teams with continued close liaison with the transplant centre [44]. 
End of life care

Adult CF centres should ensure they have the adequate skills and facilities to deal with palliative care for people with CF $[45,46]$. End of life care is ideally delivered in an adult centre where appropriate discussions and arrangements can be facilitated and the autonomy of the individual is respected and protected. This is best achieved by collaboration with specialists in palliative care though primary responsibility for patients usually remains with the CF team. Many patients now die from late complications of lung transplantation and in these cases end of life care could be provided in CF centres as well as in lung transplant units [44].

\section{Should all adults be managed in an adult CF centre?}

The maintenance of good health in childhood needs to be focused on supporting the child and the parents to ensure full engagement from the family [47]. In contrast, for the adolescent and young adults, the emphasis shifts to individual autonomy and personal responsibility of the young adult to maintain important treatments and health maintenance practices in order to preserve health. In general this requires a different set of healthcare delivery skills. The wider life experience also changes with relationships, personal development and employment becoming predominant [48]. This creates tensions and challenges due to the impact of progressive disease and requires specialised input. Increasing numbers of children progress to adulthood without chronic $P$. aeruginosa infection and with much better lung function than previously (e.g. forced expiratory volume in $1 \mathrm{~s}(\mathrm{FEV} 1)>80 \%$ predicted). This is moving most of the experience of managing difficult infections, pulmonary exacerbations and pulmonary and other complications of CF into the adult age group.

Many young adults are uncomfortable accessing care from paediatric services, which are usually family centred, developmentally focused and not appropriately geared for the culture of adult life, with the latter being focused on individual autonomy, career, education and relationship pursuit [21]. There is therefore a very strong argument for moving adult care from a paediatric environment during this time. Currently, however, this is not universally practised due to historical and operational reasons, including the absence of an appropriately trained adult teams. There are excellent examples where paediatric teams deliver whole of life care for people with CF [49-51]. However, this is not the norm in chronic diseases in most countries and is not a sustainable arrangement.

The best model considered by the task force is the establishment of a separate adult service with a referral relationship with paediatric centre(s) caring for children with CF. Some centres have a different model merging adult and paediatric services into one programme. This has some advantages in terms of sharing staff and can be an appropriate model in some circumstances. However, in this situation it is important to have appropriately trained physicians, ideally with a background in adult pulmonology as leaders for delivery of the service to adults with CF.

\section{Where should specialist adult CF centres be situated?}

Most of the mortality and morbidity in CF relates to the lung disease caused by infection and the consequent inflammatory processes which cause mucus plugging, bronchiectasis and air trapping and the associated reduction in measures of lung function such as FEV1 [52]. The medical care of adults with CF should therefore be delivered by pulmonologists who have had specific training in CF, understand the pathogenesis of CF and are aware of all of the potential interventions that will reduce the impact of infection and inflammation. The need for management of pulmonary complications, such as exacerbations, major haemoptysis, pneumothorax and respiratory failure, as well as for a clear understanding of how to prevent and manage these complications, suggests adult CF care is best based in adult pulmonology services. Other core physician team members could include infectious disease physicians, internists and gastroenterologists.

The multi-organ challenges of CF also requires access to specialists from a wide range of disciplines including, for example, hepatology, nephrology, endocrinology, particularly diabetes and metabolic bone disease, and ear, nose and throat [3,9]. Experts in reproduction, genetic counselling and obstetrics and gynaecology are also needed to deal with infertility and the management of pregnancy in CF [8]. Therefore, it is most efficient for adult CF centres to be located at large teaching/academic hospitals to support clinical care, education and training and research where appropriate support is available for all aspects of care delivery. Improving the outcomes of people with CF through the development of better treatments also requires commitment and infrastructure to support clinical research, investigator-initiated clinical trials and clinical trials sponsored by the pharmaceutical industry. The adult CF programme director should have leadership skills to support the development and sustenance of the multidisciplinary team, collaborate with paediatric and other interdisciplinary colleagues and with hospital administration; and the ability to lead a clinical research programme [21]. 


\section{What facilities should be available?}

The facilities required to deliver an adult CF service are covered in detail in the framework document in the ECFS standards of care [8]. One of the key considerations for outpatient and inpatient facilities is appropriate infection control practices. Pathogens in the CF airways can be transmitted from patient to patient, by fomites and aerosolisation in the air [53]. Adults with CF should be managed in single rooms with en suite facilities to reduce the risk of patient to patient transmission of infection. In addition, young people require privacy in managing illness and this is best delivered in a single room. Such rooms, where possible, should have appropriate ventilation to ensure high rates of air exchange and protocols should be developed for all aspects of infection control, including the management of patients in all parts of the hospital, e.g. lung function laboratory, the radiology department, pharmacy and outpatient waiting areas. The CF teams and ward nursing teams should have specific training in infection control principles as they apply to CF care. In the outpatient setting appropriate clinical space should be available. Acutely unwell patients should be able to make contact with the treating hospital for advice from CF healthcare team members ( $24 \mathrm{~h}$ per day, 7 days per week). Facilities and staffing should be available to support (where appropriate) treatment of pulmonary exacerbations in the hospital or at home [8].

\section{Is there a role for shared care for adults with CF?}

In CF paediatric care, some CF centres have shared care arrangements. The usual model is where primary responsibility for the child with $\mathrm{CF}$ is held at the CF centre, but due to practical considerations, including geography and convenience, some or all hospital visits are delivered by paediatric teams in closer proximity to the patient's home $[54,55]$. This model of care is effective when there is a clear understanding about the service to be delivered by both parties, usually as a service level agreement. Shared care also requires close communication between the local teams and the CF centre. This model is not widely applied to adult care in Europe although this may be because adult CF centres have been more recently developed and such models are uncommon in respiratory medicine. For adults, care in an adult specialist centre facilitates continuity of care and the need to respond to the many complications of CF with appropriate expertise and support services. As the population of adults with CF continues to increase, alternate models will need to be considered, especially where geographical distance from a person's home to their CF centre may be hundreds of kilometres. Shared care arrangements may be desirable, as may be the use of telemedicine directly with patients or with a local care team to enhance the ability to share care. The CF centre is responsible for developing the skills of local healthcare team members and for auditing clinical outcomes for remote patients within the centre cohort.

\section{Is there an ideal size for a CF centre?}

The optimal size of an adult CF centre has not been addressed in the scientific literature; however, according to the ECFS standards of care for CF centres, consensus is that a designated specialist adult centre should usually be a minimum of 100 patients (but not below 50 patients) and, where care is provided in smaller centres, there should be specific links with a larger centre to ensure access to appropriate multidisciplinary expertise. There is no research to indicate if there is an optimal maximum size for a CF centre. The size of adult centres will usually depend on physical and staffing constraints; however, there are currently only a few centres providing CF specialist care for more than 500 patients.

\section{What is the appropriate staffing for an adult CF centre?}

As stated earlier, the staffing for adult CF care is covered in detail in the ECFS standard of care framework document [8] and table 2 indicates the suggested full-time equivalent positions for a clinic dependent on size. As well as a minimum of two physicians to support care in adult CF centres so that continuous expertise is available, it is critically important that suitable staffing is available for multidisciplinary care, including nursing, allied health and administrative support services. To ensure sustainable service delivery, appropriate staffing levels should include leave coverage with access to training and education for the multidisciplinary team [21].

\section{How frequently should adults with CF be reviewed?}

Managing the chronic complications of CF can be very challenging for young adults and it is important that appropriate levels of input from multidisciplinary teams are available; this usually includes regular review every 2-3 months. Countries with a widely distributed population may find a solution involving connected/telehealth to provide appropriate support for some of the review visits required in CF [56-58]. Centres that have good outcomes have systems in place to regularly assess the progress of adults with CF; therefore, measures to enhance communication between patients, local healthcare providers and the $\mathrm{CF}$ centre should be strongly considered. 


\section{Conclusions}

Important progress in CF management has led to a significant increase in the number of adults with CF, which in some countries now exceeds the number of children. This higher prevalence is not matched by a concomitant increase in adult CF services throughout Europe. Considering that the number of adults is expected to increase by $75 \%$ in the next 10 years, there is a clear need to direct resources at establishing new specialised CF adult centres in major hospitals, and where possible at the level of university hospitals $[8,10]$.

Fully staffed and fully equipped adult CF centres should be the target in order to ensure a multidisciplinary approach to patient management and to reach the highest effectiveness in disease control. Low income countries, and particularly Eastern European countries, have a higher mortality in younger CF patients compared to high income countries. This is most likely to be related to the lack of established specialised services and CF multidisciplinary teams [59]. Health inequalities must be tackled to reduce the dramatic variation in the survival of people with $\mathrm{CF}$ across Europe. Implementation of best practice is mandatory to improve the management and guarantee an optimal standard of care for both paediatric and adult patients [21].

Lung disease in CF drives most clinical care requirements. The major life-threatening complications in adulthood, such as respiratory infection, respiratory failure, pneumothorax and haemoptysis, and the management of lung transplantation require expertise from trained respiratory physicians. Adult respiratory medicine is therefore the most appropriate discipline to provide medical leadership in multidisciplinary teams. The task force strongly recommends that pulmonologists who have had specific training in CF should lead the multidisciplinary teams.

The rapidly changing challenges of CF management requires that the curriculum committee keeps pace with these changes with a continuing medical education to meet the needs of healthcare workers dealing with CF. A core curriculum has to be implemented and training centres selected and accredited by the ECFS and ERS with the same modalities applied for the HERMES programme [59].

\section{References}

1 George PM, Banya W, Pareek N, et al. Improved survival at low lung function in cystic fibrosis: cohort study from 1990 to 2007. BMJ 2011; 342: d1008.

2 Simmonds NJ. Ageing in cystic fibrosis and long-term survival. Paediatr Respir Rev 2013; 14: Suppl. 1, 6-9.

3 Parkins MD, Parkins VM, Rendall JC, et al. Changing epidemiology and clinical issues arising in an ageing cystic fibrosis population. Ther Adv Respir Dis 2011; 5: 105-119.

4 Dasenbrook EC, Merlo CA, Diener-West M, et al. Persistent methicillin-resistant Staphylococcus aureus and rate of FEV1 decline in cystic fibrosis. Am J Respir Crit Care Med 2008; 178: 814-821.

5 Dasenbrook EC, Checkley W, Merlo CA, et al. Association between respiratory tract methicillin- resistant Staphylococcus aureus and survival in cystic fibrosis. JAMA 2010; 303: 2386-2392.

6 Doe SJ, McSorley A, Isalska B, et al. Patient segregation and aggressive antibiotic eradication therapy can control methicillin-resistant Staphylococcus aureus at large cystic fibrosis centres. J Cyst Fibros 2010; 9: 104-109.

7 Jennings MT, Riekert KA, Boyle MP. Update on key emerging challenges in cystic fibrosis. Med Princ Pract 2014; 23: 393-402.

8 Conway S, Balfour-Lynn IM, De Rijcke K, et al. European Cystic Fibrosis Society Standards of Care: Framework for the Cystic Fibrosis Centre. J Cyst Fibros 2014; 13: Suppl. 1, S3-S22.

9 Simmonds NJ, Macneill SJ, Cullinan P, et al. Cystic fibrosis and survival to 40 years: a case-control study. Eur Respir J 2010; 36: 1277-1283.

10 Burgel PR, Bellis G, Olesen H, et al. Future trends in cystic fibrosis demography in 34 European countries. Eur Respir J 2015; 46: 133-141.

11 McKone EF, Borowitz D, Drevinek P, et al. Long-term safety and efficacy of ivacaftor in patients with cystic fibrosis who have the Gly551Asp-CFTR mutation: a phase 3, open-label extension study (PERSIST). Lancet Respir Med 2014; 11 902-910.

12 De Boeck K, Munck A, Walker S, et al. Efficacy and safety of ivacaftor in patients with cystic fibrosis and a non-G551D gating mutation. J Cyst Fibros 2014; 13 674-680.

13 Wainwright CE. Ivacaftor for patients with cystic fibrosis. Expert Rev Respir Med 2014; 8: 533-538.

14 Ramsey BW, Davies J, McElvaney NG, et al. A CFTR potentiator in patients with cystic fibrosis and the G551D mutation. N Engl J Med 2011; 365: 1663-1672.

15 Plant BJ, Goss CH, Plant WD, et al. Management of comorbidities in older patients with cystic fibrosis. Lancet Respir Med 2013; 1: 164-174.

16 Roehmel JF, Schwarz C, Mehl A, et al. Hypersensitivity to antibiotics in patients with cystic fibrosis. J Cyst Fibros 2014; 13: 205-211.

17 Gory I, Brown G, Wilson J, et al. Increased risk of colorectal neoplasia in adult patients with cystic fibrosis: a matched case-control study. Scand J Gastroenterol 2014; 49: 1230-1236.

18 Maisonneuve P, Marshall BC, Knapp EA, et al. Cancer risk in cystic fibrosis: a 20-year nationwide study from the United States. J Natl Cancer Inst 2013; 105: 122-129.

19 Hanna RM, Weiner DJ. Overweight and obesity in patients with cystic fibrosis: a center-based analysis. Pediatr Pulmonol 2015; 50: 35-44.

20 Buehler T, Steinmann M, Singer F, et al. Increased arterial stiffness in children with cystic fibrosis. Eur Respir J 2012; 39: 1536-1537. 
21 Smyth AR, Bell SC, Bojcin S, et al. European Cystic Fibrosis Society Standards of Care: Best Practice guidelines. J Cyst Fibros 2014; 13: Suppl. 1, S23-S42.

22 Stern M, Bertrand DP, Bignamini E, et al. European Cystic Fibrosis Society Standards of Care: Quality Management in cystic fibrosis. J Cyst Fibros 2014; 13: Suppl. 1, S43-S59.

23 Tuchman L, Schwartz M. Health outcomes associated with transition from pediatric to adult cystic fibrosis care. Pediatrics 2013; 132: 847.

24 Nasr SZ. Cystic fibrosis in adolescents and young adults. Adolesc Med 2000; 11: 589-603.

25 Arias Llorente RP, Bousono Garcia C, Diaz Martin JJ. Treatment compliance in children and adults with cystic fibrosis. J Cyst Fibros 2008; 7: 359-367.

26 O'Donohoe R, Fullen BM. Adherence of subjects with cystic fibrosis to their home program: a systematic review. Respir Care 2014; 59: 1731-1746.

27 Sawicki GS, Heller KS, Demars N, et al. Motivating adherence among adolescents with cystic fibrosis: youth and parent perspectives. Pediatr Pulmonol 2015; 50: 127-136.

28 Quittner AL, Goldbeck L, Abbott J, et al. Prevalence of depression and anxiety in patients with cystic fibrosis and parent caregivers: results of The International Depression Epidemiological Study across nine countries. Thorax 2014; 69: 1090-1097.

29 Bolyard DR. Sexuality and cystic fibrosis. MCN Am J Matern Child Nurs 2001; 26: 39-41.

30 Cohen-Cymberknoh M, Shoseyov D, Kerem E. Managing cystic fibrosis: strategies that increase life expectancy and improve quality of life. Am J Respir Crit Care Med 2011; 183: 1463-1471.

31 Sawicki GS, Ren CL, Konstan MW, et al. Treatment complexity in cystic fibrosis: trends over time and associations with site-specific outcomes. J Cyst Fibros 2013; 12: 461-467.

32 Quittner AL, Zhang J, Marynchenko M, et al. Pulmonary medication adherence and health-care use in cystic fibrosis. Chest 2014; 146: 142-151.

33 Duff AJ, Latchford GJ. Motivational interviewing for adherence problems in cystic fibrosis. Pediatr Pulmonol 2010; 45: 211-220.

34 Smith B, Goldbeck L, Georgiopoulos AM. Screening and assessment: psycological intervention, pharmacologicial interventions. Ped Pulmonol 2014; 49: 171-172.

35 Ernst MM, Johnson MC, Stark LJ. Developmental and psychosocial issues in cystic fibrosis. Pediatr Clin North Am 2011; 58: 865-885.

36 Goldbeck L, Fidika A, Herle M, et al. Psychological interventions for individuals with cystic fibrosis and their families. Cochrane Database Syst Rev 2014; 6: CD003148.

37 Dill EJ, Dawson R, Sellers DE, et al. Longitudinal trends in health-related quality of life in adults with cystic fibrosis. Chest 2013; 144: 981-989.

38 Schram CA. Atypical cystic fibrosis: identification in the primary care setting. Can Fam Physician 2012; 58: 1341-1345.

39 Bombieri C, Claustres M, De Boeck K, et al. Recommendations for the classification of diseases as CFTR-related disorders. J Cyst Fibros 2011; 10: Suppl. 2, S86-102.

40 Corris PA. Lung transplantation for cystic fibrosis and bronchiectasis. Semin Respir Crit Care Med 2013; 34: 297-304.

41 Mayer-Hamblett N, Rosenfeld M, Emerson J, et al. Developing cystic fibrosis lung transplant referral criteria using predictors of 2-year mortality. Am J Respir Crit Care Med 2002; 166: 1550-1555.

42 Lucangelo U, Del Sorbo L, Boffini M, et al. Protective ventilation for lung transplantation. Curr Opin Anaesthesiol 2012; 25: 170-174.

43 Gattinoni L, Carlesso E, Langer T. Clinical review: extracorporeal membrane oxygenation. Crit Care 2011; 15: 243.

44 Hirche TO, Knoop C, Hebestreit $\mathrm{H}$, et al. Practical guidelines: lung transplantation in patients with cystic fibrosis. Pulm Med 2014; 2014: 621342.

45 Braithwaite M, Philip J, Tranberg H, et al. End of life care in CF: patients, families and staff experiences and unmet needs. J Cyst Fibros 2011; 10: 253-257.

46 Sands D, Repetto T, Dupont LJ, et al. End of life care for patients with cystic fibrosis. J Cyst Fibros 2011; 10: S37-S44.

47 McDonald CM, Haberman D, Brown N. Self-efficacy: empowering parents of children with cystic fibrosis. J Cyst Fibros 2013; 12: 538-543.

48 Taylor-Robinson DC, Smyth R, Diggle PJ, et al. A longitudinal study of the impact of social deprivation and disease severity on employment status in the UK cystic fibrosis population. PLoS One 2013; 8: e73322.

49 Gleeson H, Turner G. Transition to adult services. Arch Dis Child Educ Pract Ed 2012; 97: 86-92.

50 Madge S, Bryon M. A model for transition from pediatric to adult care of care in cystic fibrosis. J Pediatr Nurs 2002; 17: 283-288.

51 Flume PA, Taylor LA, Anderson DL, et al. Transition programs in cystic fibrosis centers: perceptions of team members. Pediatr Pulmonol 2004; 37: 4-7.

52 Dassios T. Determinants of respiratory pump function in patients with cystic fibrosis. Paediatr Respir Rev 2015; 16: 75-79.

53 Saiman L, Siegel JD, LiPuma JJ, et al. Infection prevention and control guideline for cystic fibrosis: 2013 update. Infect Control Hosp Epidemiol 2014; 35: Suppl. 1, S1-S67.

54 Doull I, Evans H, South and Mid Wales Paediatric Cystic Fibrosis Network. Full, shared and hybrid paediatric care for cystic fibrosis in South and Mid Wales. Arch Dis Child 2012; 97: 17-20.

55 Elborn JS, Hodson M, Bertram C. Implementation of European standards of care for cystic fibrosis - provision of care. J Cyst Fibros 2009; 8: 348-355.

56 Bella S, Murgia F, Cotognini C, et al. Program of home telemonitoring in patients with cystic fibrosis over a period of 2 years: a contribution to the rationalization of care. Clin Ter 2013; 164: e313-e317.

57 Cox NS, Alison JA, Rasekaba T, et al. Telehealth in cystic fibrosis: a systematic review. J Telemed Telecare 2012; 18: 72-78.

58 Bella S, Murgia F, Cotognini C, et al. Program of home telemonitoring in patients with cystic fibrosis over a period of 2 years: a contribution to the rationalization of care. Clin Ter 2013; 164: e313-e317.

59 Harmonised Education in Respiratory Medicine for European Specialists. Accreditation process. http://hermes. ersnet.org/accreditation/accreditation-process/ 